\title{
Hypolipidernic Effect of Gemfibrozil in Stroke-Prone Spontaneously Hypertensive Rat (SHRSP) Fed a High-Fat and High-Cholesterol Diet.
}

Hiroshi Ogawa ${ }^{1}$, Masatsuna Tasaka ${ }^{1}$ and Sukanari Sasagawa ${ }^{2}$. ${ }^{1}$ Department of Hygiene, Kinki University School of Medicine, Osaka 589, ${ }^{2}$ Research Institute of Life Sciences, Kinki University, Osaka 589 JAPAN.

In the preceding paper, we have reported the effect of gemfibrozil (Lopid) on lipid metabolism, using stroke-prone SHR (SHRSP) as an animal model. In SHRSP fed a regular diet, gemfibrozil decreases serum level of very low density lipoprotein (VLDL) and increases serum level of high density lipoprotein (HDL) rich in apoE (apoE-HDL), while it has no effect on blood pressure. These changes in lipoprotein metabolism of SHRSP suggest that gemfibrozil exerts antiatherogenic and hypolipidemic actions in the hyperlipidemic SHRSP as in the case reported by Krause et al. (J LIPID RES 26: 940, 1985).

The purpose of this study is to investigate effects of gemfibrozil on lipid metabolism in the hyperlipidemic SHRSP induced by a high-fat and high-cholesterol diet (HFC diet) feeding.

\section{MATERIAL AND METHODS:}

Seven-week-old male SHRSPs were used and divided into two groups. Control group was given a HFC diet prepared by adding $3 \%$ lard, $1.2 \%$ cholesterol and $0.3 \%$ cholic acid to a regular diet (CE-2, Clea Japan Inc.) by weight. Experimental group was given the control (HFC) diet containing $0.05 \%$ gemfibrozil. The duration of the experiment was 4 weeks with free access to the diet and drinking water. On day 21 , post-heparin plasma was obtained from the jugular vein under ether anesthesia without fasting and the lipolitic activities were measured by immunoprecipitationRI method as reported previously. On day 28 , whole blood was obtained from the abdominal aorta under ether anesthesia after fasting overnight, and the liver was immediately excised. Serum was separated by low centrifugation and serum lipoprotein fractions were isolated by stepwisely density-gradient ultracentrifugation. Lipid contents in the serum, lipoprotein fractions and the liver were determined by the same method as reported previously. The contents of apolipoproteins were estimated by electroimmunoassay. The activities of hepatic cholesterol $7 \alpha$-hydroxylase $(7 \alpha$ hydroxylase) and acyl-CoA:cholesterol acyltransferase (ACAT) were measured by HPLC method.

\section{RESULTS AND DISCUSSION:}

There was no significant difference between the two groups in the growth rate and food intake. There was no significant change in blood pressure. Gemfibrozil dramatically reduced serum total cholesterol (TC) level by $53.6 \%$, and significantly reduced serum phospholipid (PL) and triglyceride (TG) levels by $20 \%$ and $38.4 \%$, respectively. In addition, gemfibrozil significantly raised serum concentrations of apoA-I, A-IV and E by $112 \%, 27.5 \%$ and $42.5 \%$, respectively, and significantly reduced senum concentration of apoB by $36.7 \%$. The marked decreases in serum TC, PL and TG levels were mainly due to the decreases in cholesterol-rich $\beta$-VLDL and low density lipoprotein (LDL), judging from lipid distributions among various lipoprotein fractions. In contrast, there was no significant increase in HDL-cholesterol level, although apoA-I and A-IV contents in the HDL fraction were significantly increased by gemfibrozil treatment. This suggests the change in HDL composition, in other words, the increases in HDL subfractions containing apoA-I and/or apoA-IV. These changes in serum lipid metabolism were associated with changes in the activities of lipoprotein lipase and hepatic triglyceride lipase .

In the liver, the contents of TC, PL and TG markedly decreased by $74.5 \%, 23.9 \%$ and $63.9 \%$, respectively, which was associated with a tendency of both an increase in $7 \alpha$-hydroxylase activity and a decrease in ACAT activity. These results suggest that gemfibrozil shows a effective lipidlowering action in the liver of the hyperlipidemic SHRSP. 